\title{
Pigmented Corneal Ulcer
}

\author{
Sonam Yangzes, MS; Parul Chawla Gupta, MS; Vivek Jha, MS; Jagat Ram, MS, FAMS \\ Department of Ophthalmology, Post Graduate Institute of Medical Education and Research, Chandigarh, India \\ ORCID: \\ Sonam Yangzes: https://orcid.org/0000-0002-1862-0254 \\ Jagat Ram: https://orcid.org/0000-00016251-2800
}

\begin{abstract}
Purpose: To report the clinical characteristics, laboratory findings, and treatment of a rare case of keratitis caused by pigmented fungi Bipolaris hawaiiensis.

Case Report: A 55-year-old man presented with a history of trauma with vegetative matter in his left eye. Slit lamp biomicroscopic examination revealed the presence of a brownish-black pigmented plaque with surrounding infiltrates. Corneal scrapings revealed multiple septate hyphae. Culture revealed growth of the Bipolaris species. The patient was treated with topical natamycin $5 \%$, topical voriconazole $1 \%$, and oral itraconazole followed by intracameral amphotericin $\mathrm{B}(5 \mu \mathrm{g} / \mathrm{mL})$. The patient responded well to the treatment. Conclusion: Brown pigmented infiltrates are an important clinical feature of dematiaceous fungi. $B$. hawaiiensis is a rare cause of corneal phaeohyphomycosis. Our patient responded well to intracameral amphotericin B, which obviated the need for penetrating keratoplasty.
\end{abstract}

Keywords: Corneal Ulcer; Keratitis; Pigmented

J Ophthalmic Vis Res 2019; 14 (4): 506-508

Correspondence to:

Jagat Ram, MS, FAMS. Department of Ophthalmology, Post Graduate Institute of Medical Education and Research, Chandigarh 160012, India.

E-mail: drjagatram@gmail.com

Received: 15-05-2018Ａccepted: 09-09-2018

Access this article online

Website:

https://knepublishing.com/index.php/JOVR

DOI:

10.18502/jovr.v14i4.5461

\section{INTRODUCTION}

Fungal keratitis is one of the most common causes of keratitis in tropical countries. ${ }^{[1,2]}$ Dematiaceous fungi are the third most common cause of keratomycosis, with Curvularia and Bipolaris being the most common infecting species. ${ }^{[3,4]}$

Bipolaris hawaiiensis is a darkly pigmented fungus, widely distributed in nature, that causes cutaneous and soft tissue diseases known as phaeohyphomycosis; ${ }^{[5]}$ it is an extremely uncommon cause of keratitis. Oral itraconazole and topical natamycin have been used for this condition, while a few case reports have described additional use of topical amphotericin B in refractory cases. We report a case of keratomycosis, caused by $B$. hawaiiensis, that was treated with intracameral amphotericin B and showed a good response.

\section{CASE REPORT}

A 55-year-old male farmer with a history of trauma to the left eye with vegetative matter presented at the ophthalmology clinic with complaints of diminution of vision, redness, photophobia, and black discoloration of his left eye. The visual acuity

This is an open access journal, and articles are distributed under the terms of the Creative Commons Attribution-NonCommercial-ShareAlike 4.0 License, which allows others to remix, tweak, and build upon the work non-commercially, as long as appropriate credit is given and the new creations are licensed under the identical terms.

How to cite this article: Yangzes S, Gupta PC, Jha V, Ram J. Pigmented Corneal Ulcer. J Ophthalmic Vis Res 2019;14:506-508. 


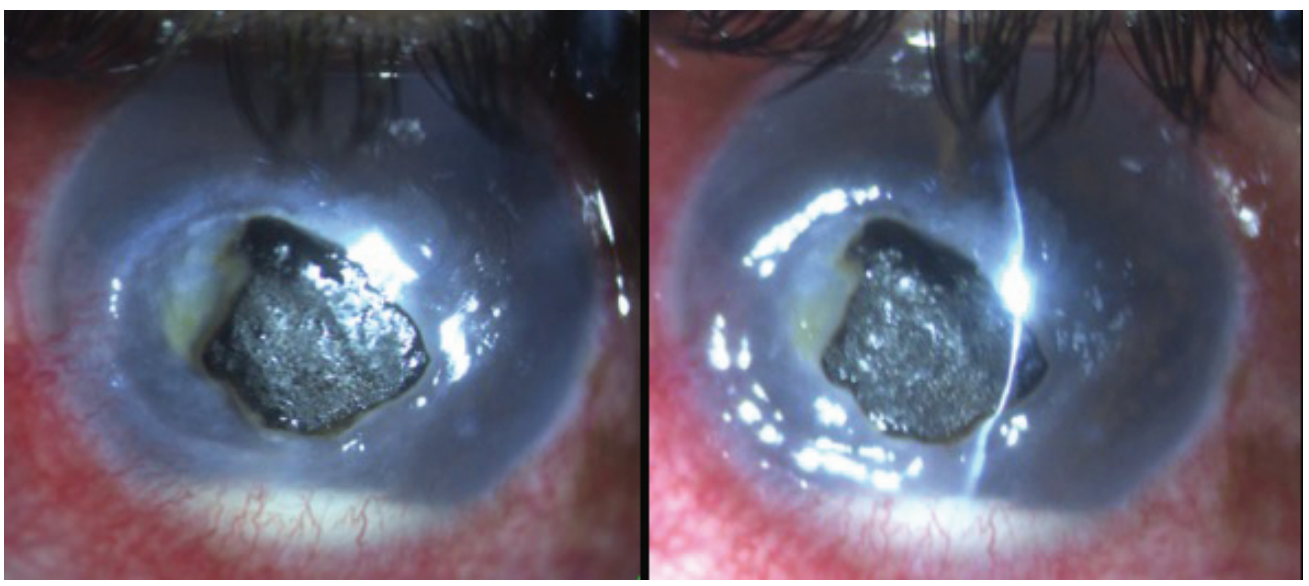

Figure 1. (a) A corneal ulcer measuring $6.5 \times 5.5 \mathrm{~mm}$, with a central pigmented plaque $4 \times 4 \mathrm{~mm}$ with hypopyon. (b) Slit view showing area of corneal thinning.

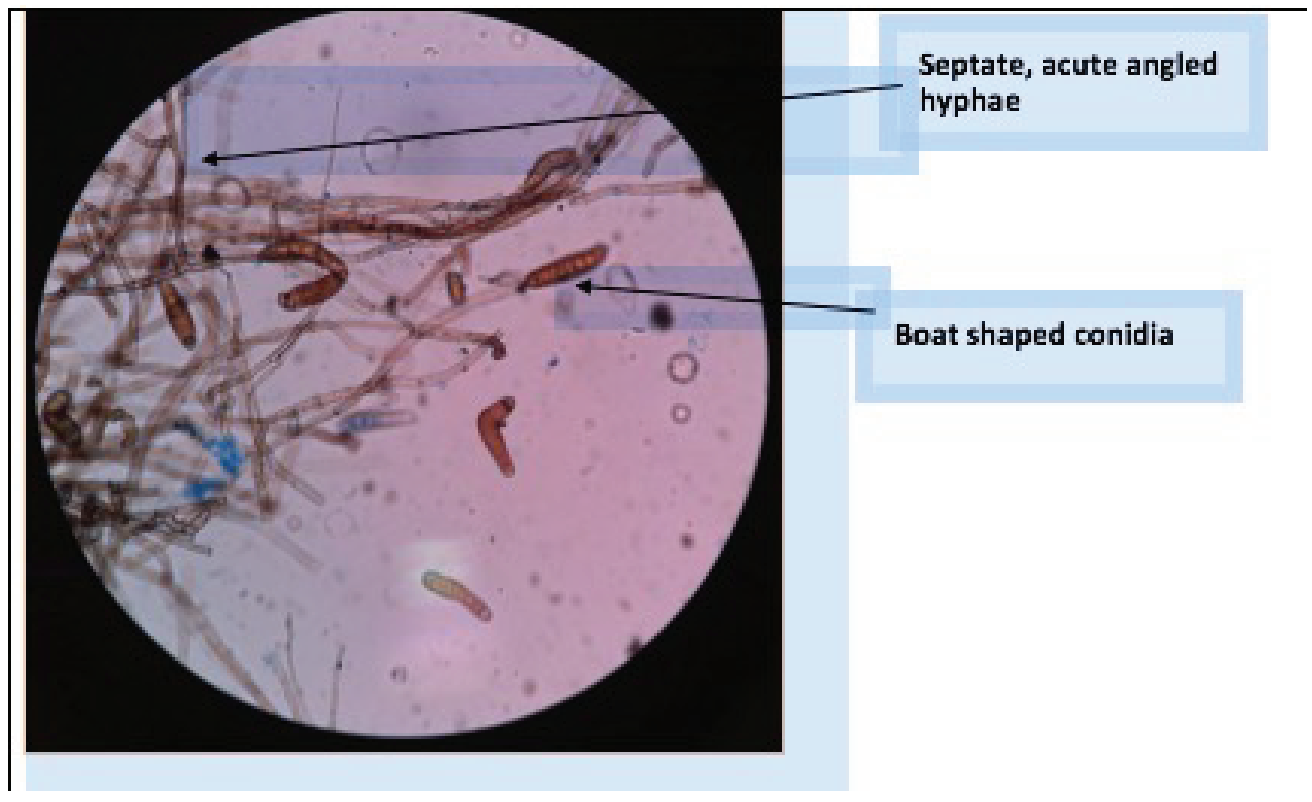

Figure 2. Lactophenol cotton blue staining showing acute angled septate hyphae with boat-shaped conidia.

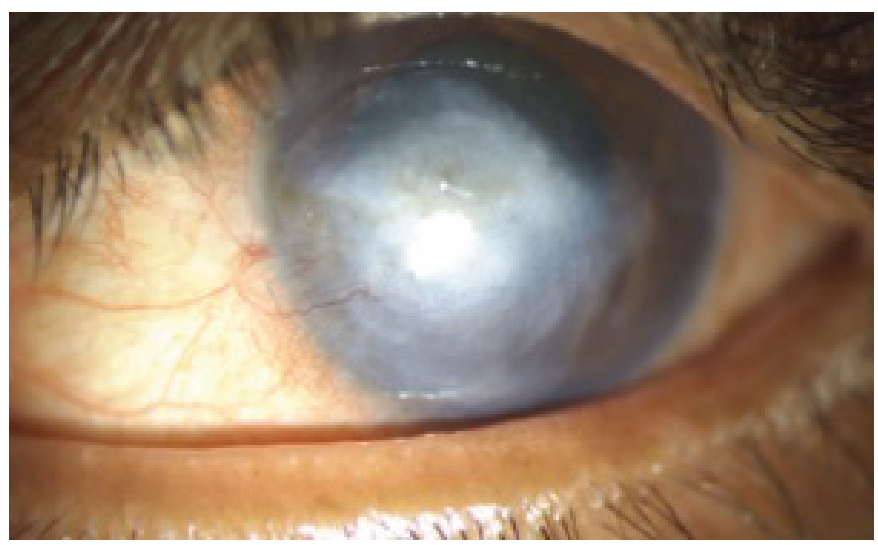

Figure 3. Healed ulcer with central scarring and peripheral vascularization. 
in the right eye was $6 / 6$, while vision in the affected eye was restricted to the perception of light.

Slit lamp biomicroscopy revealed conjunctival congestion, a central pigmented corneal ulcer, and hypopyon. There were no other predisposing factors such as diabetes or other immunocompromised states. The central ulcer measured 6.5 x $5.5 \mathrm{~mm}$, and showed a pigmented, elevated brownish-black plaque measuring $4 \times 4 \mathrm{~mm}$, with surrounding infiltrates. The ulcer appeared dry, with the presence of immobile hypopyon [Figures $1(a)$ and (b)]. The right eye presented with a clear cornea, early cataract, and a normal fundus. The plaque was scraped off and used to inoculate culture media. Due to the typical appearance of the ulcer (dry, pigmented plaque), we initiated hourly topical antifungal natamycin $5 \%$ treatment along with two-hourly topical moxifloxacin $0.5 \%$, and cycloplegic and lubricating eyedrops. The culture revealed growth of the Bipolaris species. Lactophenol cotton blue staining showed acuteangled septate hyphae with boat-shaped conidia [Figure 2]. The hypopyon remained refractory to treatment after which amphotericin B $(5 \mu \mathrm{g} / \mathrm{mL})$ was injected intracamerally. The infiltrates became organized and the hypopyon disappeared. The lesion healed completely with central scarring and vascularization [Figure 3]. The final visual acuity was finger counting close to the face with accurate projection of light.

\section{DISCUSSION}

The Bipolaris species is classified as a dematiaceous or darkly pigmented fungus that causes phaeohyphomycosis, rarely infecting humans. The most frequently reported species are $B$. spicifera, $B$. australiensis, and B. hawaiiensis. ${ }^{[6]}$ Anadi et al have reported corneal ulcers due to Bipolaris spp. infection in a leprosy patient, ${ }^{[7]}$ while Bashir et al have reported keratomycosis with endophthalmitis in an immunocompetent individual. ${ }^{[8]} B$. hawaiiensis is widely distributed in plants or soil and is commonly found in tropical regions. Dematiaceous fungal keratitis presents typically as recalcitrantpigmented plaques that prevent the penetration of drugs, making superficial keratectomy essential in many cases. ${ }^{[9]}$ Culture shows dark septate hyphae with numerous conidia of the Bipolaris species. Although, the use of topical natamycin has been reported to be successful in treating most cases of dematiaceous fungal keratitis, ${ }^{[3]}$ our case responded well to intracameral amphotericin $B$ injection, which obviated the need for long-term use of oral antifungal drugs as well as therapeutic penetrating keratoplasty.

\section{Financial Support and Sponsorship}

Nil.

\section{Conflicts of Interest}

There are no conflicts of interest.

\section{REFERENCES}

1. Chowdhary A, Singh K. Spectrum of fungal keratitis in North India. Cornea 2005;24:8-15.

2. Tanure MA, Cohen EJ, Sudesh S, Rupuano CJ, Laibson PR. Spectrum of fungal keratitis at Wills Eye Hospital, Philadelphia, Pennsylvania. Cornea 2000;19:307-312.

3. Garg P, Gopinathan U, Chaudhary K, Rao GN. Keratomycosis: clinical and microbiologic experience with dematiaceous fungi. Ophthalmology 2000;107:574-580.

4. Gopinathan U, Garg P, Fernandes M, Sharma S, Athmanathan S, Rao GN. The epidemiological and laboratory results of fungal keratitis: a 10 year review at referral eye care center in South India. Cornea 2002;21:555-559.

5. Ajello L, Georg LK, Steigbigel RT, Wang CJ. A case of phaeohyphomycosis caused by a new species of phialophora. Mycologia 1974;66:490-498.

6. Cunha KC, Sutton DA, Fothergill AW, Cano J, Gené J, Madrid $\mathrm{H}$, et al. Diversity of Bipolaris species in clinical samples in the United States and their antifungal susceptibility profiles. J Clin Microbio/ 2012;50:4061-4066.

7. Anandi V, Suryawanshi NB, Koshi G, Padhye AA, Ajello L. Corneal ulcer caused by Bipolaris hawaiiensis. J Med Vet Mycol 1988;26:301-306.

8. Bashir G, Hussain W, Rizvi A. Bipolaris hawaiiensis keratomycosis and endophthalmitis. Mycopathologia 2009;167:51-53.

9. Garg P, Vemuganti GK, Chatarjee S, Gopinathan U, Rao GN. Pigmented plaque presentation of Dematiaceous fungal keratitis: a Clinicopathologic Correlation. Cornea 2004;23:57-576. 